\title{
Dual-wavelength high-power diode laser system based on an external-cavity tapered amplifier with tunable frequency difference
}

\author{
Chi, Mingjun; Jensen, Ole Bjarlin; Petersen, Paul Michael
}

Published in:

Optical Society of America. Journal B: Optical Physics

Link to article, DOI:

10.1364/JOSAB.29.002617

Publication date:

2012

Document Version

Publisher's PDF, also known as Version of record

Link back to DTU Orbit

Citation (APA):

Chi, M., Jensen, O. B., \& Petersen, P. M. (2012). Dual-wavelength high-power diode laser system based on an external-cavity tapered amplifier with tunable frequency difference. Optical Society of America. Journal B: Optical Physics, 29(9), 2617-2621. https://doi.org/10.1364/JOSAB.29.002617

\section{General rights}

Copyright and moral rights for the publications made accessible in the public portal are retained by the authors and/or other copyright owners and it is a condition of accessing publications that users recognise and abide by the legal requirements associated with these rights.

- Users may download and print one copy of any publication from the public portal for the purpose of private study or research.

- You may not further distribute the material or use it for any profit-making activity or commercial gain

- You may freely distribute the URL identifying the publication in the public portal 


\title{
Dual-wavelength high-power diode laser system based on an external-cavity tapered amplifier with tunable frequency difference
}

\author{
Mingjun Chi,* Ole Bjarlin Jensen, and Paul Michael Petersen \\ DTU Fotonik, Department of Photonics Engineering, Technical University of Denmark, Frederiksborgvej 399, \\ P.O. Box 49, DK-4000 Roskilde, Denmark \\ ${ }^{*}$ Corresponding author: mchi@fotonik.dtu.dk
}

Received May 9, 2012; revised July 19, 2012; accepted July 19, 2012;

posted July 20, 2012 (Doc. ID 168331); published August 30, 2012

\begin{abstract}
A dual-wavelength high-power semiconductor laser system based on a tapered amplifier with double-Littrow external cavity is demonstrated around $800 \mathrm{~nm}$. The two wavelengths can be tuned individually, and the frequency difference of the two wavelengths is tunable from 0.5 to $10.0 \mathrm{THz}$. To our knowledge, this is the broadest tuning range of the frequency difference from a dual-wavelength diode laser system. The spectrum, output power, and beam quality of the diode laser system are characterized. The power stability of each wavelength is measured, and the power fluctuations of the two wavelengths are almost of opposite phase. The simultaneous emission of the two wavelengths is verified by a sum-frequency generation experiment in a bismuth triborate nonlinear crystal. @ 2012 Optical Society of America
\end{abstract}

OCIS codes: $\quad 140.0140,140.5960,140.3280$

\section{INTRODUCTION}

Dual-wavelength diode laser systems are attractive for many applications, such as dual-wavelength interferometry, optical switching, terahertz $(\mathrm{THz})$ radiation generation, and $\mathrm{THz}$ imaging [1-5]. Different techniques have been developed to achieve dual-wavelength operation of a diode laser system, and they mainly can be classified into two categories: (1) Monolithic dual-wavelength diode lasers [6-10], and (2) diode laser systems based on different external-cavity feedback techniques [11-17]. The monolithic dual-wavelength diode lasers show stable dual-wavelength operation, but the frequency difference of the two wavelengths is not tunable or the tuning range is limited, and the output power from the monolithic dual-wavelength diode lasers is normally less than $500 \mathrm{~mW}$ [8]. Different external-cavity feedback techniques have been developed for dual-wavelength diode laser systems, and different frequency selective elements have been used in these dual-wavelength external-cavity diode laser systems, such as bulk diffractive gratings for the double-Littman and double-Littrow external-cavity techniques [11, $\underline{12}, \underline{15}]$, dual-fiber Bragg grating [13], dual-period holographic element [14], and single-wavelength volume Bragg gratings or monolithic multiplexed Bragg grating [16,17].

The gain device in the external-cavity dual-wavelength diode laser system is usually a single-mode ridge-waveguide diode laser, so the output power from these laser systems is normally a few hundreds milliwatts [11-15]. In a few cases, broad-area diode lasers are used as gain device, and more than $1.7 \mathrm{~W}$ output power has been achieved with fixed frequency difference $[\underline{16}, \underline{17}]$. Due to the broad emitting aperture of broad-area diode lasers in the slow axis, these dualwavelength diode laser systems suffer from poor beam quality in the slow axis. Recently, we demonstrated the first dualwavelength external-cavity diode laser system based on a tapered diode amplifier with tunable THz frequency difference [18]. More than $1.5 \mathrm{~W}$ output power was obtained and the frequency difference of the two wavelengths was tunable up to $5.0 \mathrm{THz}$.

Tapered diode amplifiers consist of an index-guided ridgewaveguide section and a gain-guided tapered section. The ridge-waveguide section works as a spatial filter in the slow axis, thus diffraction-limited laser beam is available from tapered diode amplifier in both fast and slow axes. High output power (depending on the wavelength from a few watts to 10 watts) can be obtained due to the amplification from the gain-guided tapered section [19].

In this paper, we present a new improved dual-wavelength diode laser system based on the tapered amplifier. Compared with the previous system, it is easier to make the new laser system oscillate in the dual-wavelength mode. The frequency difference of the two wavelengths is tunable from 0.5 to 10.0 THz. The suppression of amplified spontaneous emission intensity is more than $25 \mathrm{~dB}$ in the tuning range of the frequency difference. An output power of $1.6 \mathrm{~W}$ is obtained with a frequency difference of $0.57 \mathrm{THz}$, and the output power is higher than $0.95 \mathrm{~W}$ in the entire tuning range of frequency difference. The beam quality factor $M^{2}$ in the slow axis is $1.24 \pm 0.12$ at an output power of $1.45 \mathrm{~W}$. The output power fluctuation of the two wavelengths are of opposite phase, thus the fluctuation of the total power is much less than that of each of the two wavelengths. We demonstrated the sumfrequency generation (SFG) of the two wavelengths from the dual-wavelength diode laser system in a bismuth triborate (BIBO) crystal. This is an experimental verification of 
simultaneous emission of the two wavelengths from the laser system, although the quantum well is normally realized as a homogeneous-broaden gain medium.

\section{EXPERIMENTAL SETUP OF THE DUAL- WAVELENGTH DIODE LASER SYSTEM}

In the dual-wavelength diode laser system, an $800 \mathrm{~nm}$ tapered amplifier is used as a gain medium. The epitaxial structure of the $800 \mathrm{~nm}$ tapered amplifier was grown using metal organic vapor phase epitaxy. A tensile strained single GaAsP quantum well was used as gain medium, which was embedded in an AlGaAs waveguide. The side view of the geometry of the tapered amplifier in the experimental setup is shown in Fig. 1(a). The processed tapered gain device consisted of a $1 \mathrm{~mm}$ long index-guided ridge-waveguide section and a $3 \mathrm{~mm}$ long flared section. The width of the ridge-waveguide section is around $3 \mu \mathrm{m}$. The tapered angle was $4^{\circ}$, and the output aperture was $210 \mu \mathrm{m}$. The rear facet was antireflection coated with a reflectivity of $0.05 \%$, while the front facet had a reflectivity of $0.5 \%$.

The top view of the double-Littrow external-cavity configuration applied in the experiment is depicted in Fig. 1(b). An aspherical lens $\left(L_{1}\right)$ of $3.1 \mathrm{~mm}$ focal length with a numerical aperture (NA) of 0.68 is used to collimate the beam from the rear facet in both fast and slow axes. The collimated beam is split into two beams by a polarizing beam splitter (PBS) after passing through a half-wave plate (HWP). Each beam is incident on a bulk diffraction grating, which is ruled with 1200 grooves $/ \mathrm{mm}$ and has a blazed wavelength of $750 \mathrm{~nm}$. The gratings are mounted in the Littrow configuration and oriented with the lines in the grating parallel to the slow axis direction. A second aspherical lens $\left(L_{2}\right)$ of $3.1 \mathrm{~mm}$ focal length with an NA of 0.68 is used to collimate the beam from the front facet in the fast axis. Together with the aspherical lens, a cylindrical lens $\left(L_{3}\right)$ of $50 \mathrm{~mm}$ focal length is used to collimate the beam in the slow axis and compensate for the astigmatism in the tapered amplifier. A beam splitter (BS) is used to reflect part of the output beam of the diode laser system as a diagnostic beam, where the spectrum and beam quality factor $M^{2}$ of the output beam are measured. The output power of the

(a)

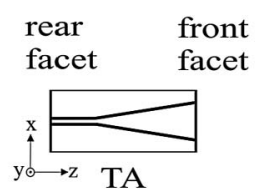

(b)

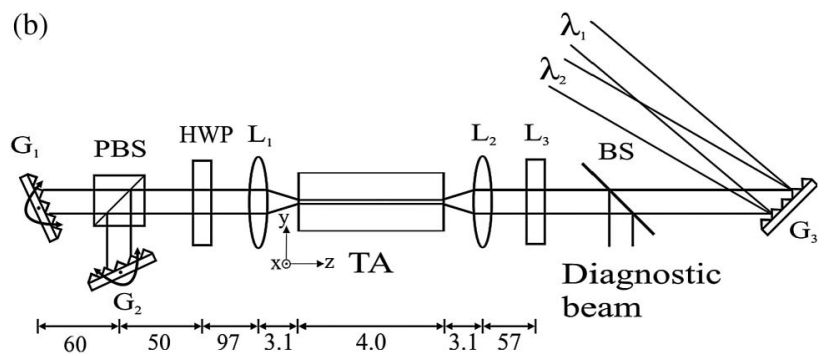

Fig. 1. (a) Side view of the tapered diode laser amplifier in the experimental setup. (b) Top view of the experimental setup of the dual-wavelength diode laser system. TA, tapered amplifier; $L_{1}, L_{2}$, aspherical lenses; $L_{3}$, cylindrical lens; PBS, polarized beam splitter; BS, beam splitter; HWP, half-wave plate; $G_{1}, G_{2}, G_{3}$, diffraction gratings; $\lambda_{1}, \lambda_{2}$, wavelength 1 and 2 . The units are in $\mathrm{mm}$. laser system is measured behind the second aspherical lens. In order to measure the stability of the output power in each wavelength, a third grating $\left(G_{3}\right.$, ruled with 1200 grooves $/ \mathrm{mm}$ and with a blazed wavelength of $750 \mathrm{~nm}$ ) is used to disperse the dual-wavelength output beam into two monochromatic beams with different propagating directions. All the lenses, HWP, and BSs are antireflection coated for the near-infrared wavelength. The temperature of the tapered amplifier is controlled with a Peltier element and it is operated at $25{ }^{\circ} \mathrm{C}$ in the experiment.

The polarization direction of the output beam is measured, and the ratio of the powers polarized along the fast and slow axes is $100: 1.1$; this means the TM mode in the tapered amplifier dominates the laser emission of the diode laser system. So the output beam from the rear facet is almost linearly polarized along the fast axis. After the HWP, the polarization direction of the laser beam is rotated to around $45^{\circ}$ to the fast axis. The output beam is split by the PBS into two beams, one beam polarized along the fast axis and the other polarized along the slow axis, and the powers of these two beams are almost equal. The beam polarized along the fast axis is directed to grating $G_{1}$, and the beam polarized along the slow axis is directed to grating $G_{2}$. The gratings are mounted in the Littrow configuration, this means the first-order diffraction beams of the gratings propagate back toward the tapered amplifier. Therefore, the laser cavities are formed between the gratings and the front facet of the tapered amplifier. The tapered amplifier works as a gain medium in the laser cavity. The emission wavelengths $\lambda_{1}$ (from grating $G_{1}$ ) and $\lambda_{2}$ (from grating $G_{2}$ ) of the laser system are tuned by rotating the gratings in the $Y Z$ plane [as shown in Fig. 1(b)] independently.

The difference between the present and the previous dualwavelength diode laser systems is: instead of a cube 50/50 BS in the previous setup, a combination of a HWP and a PBS is used in the present setup to split the output beam from the rear facet into two beams, and each of the beams is incident on a bulk diffraction grating. The advantage of this change is: the ratio of the powers directed to the two gratings can be adjusted by rotating the HWP, i.e., the relative feedback strengths from the two arms can be controlled. Two factors determine if the two wavelengths from the diode laser system oscillate simultaneously: the gain of each wavelength and the feedback strength of each wavelength. In the experiment, we rotate the two gratings in the $Y Z$ plane to find two wavelengths with the gains as close as possible. But normally a dual-wavelength operation from the diode laser system is not achievable by tuning the two wavelengths only. To obtain a dual-wavelength operation, the feedback strengths of the two wavelengths have to be adjusted to compensate for the possible gain difference of the two wavelengths. In the previous diode laser system [18], the feedback strengths of the two wavelengths were adjusted by rotating the diffraction gratings in the $X Z$ plane, i.e., adjusting the coupling efficiency of the laser beams into the tapered device. This process is complex and takes a long time since the two gratings have to be adjusted in both $Y Z$ and $X Z$ planes. In the present setup, the feedback strengths are adjusted by rotating the HWP. Thus, it is easier to make the laser system oscillate in dualwavelength mode compared with the previous system [18]. Here we should mention that the dual-wavelength operation is quite sensitive to the relative feedback strengths of the two 


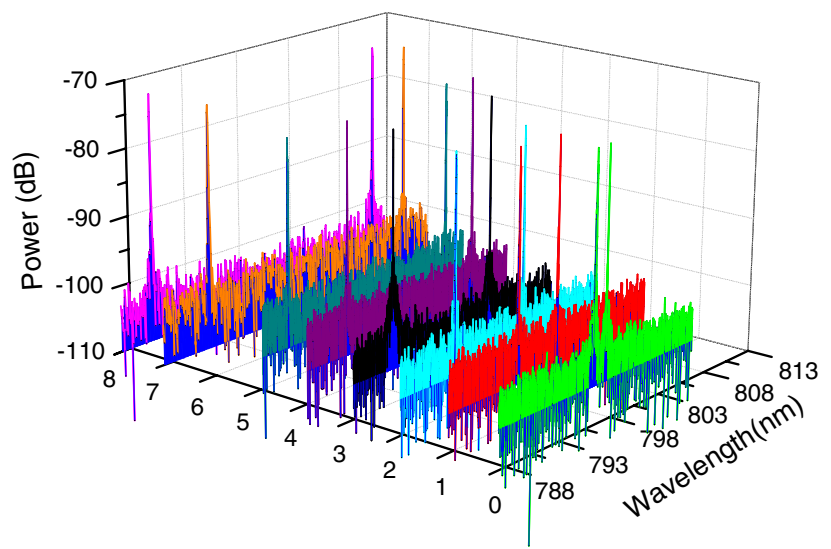

Fig. 2. (Color online) Optical spectra of the output beam from the dual-wavelength tapered diode laser system with frequency difference from 0.57 to $10.02 \mathrm{THz}$. The operating current is $3.5 \mathrm{~A}$.

wavelengths, and it is more sensitive at the low frequency end of the frequency difference. A rotation of the HWP of half degree will terminate a stable dual-wavelength operation.

\section{EXPERIMENTAL RESULTS OF THE DUAL- WAVELENGTH DIODE LASER SYSTEM}

The output power at different wavelengths for the diode laser system based on the same tapered amplifier with singleLittrow external-cavity feedback [20] was measured at an operating current of $3.5 \mathrm{~A}$ [18]. According to the tuning curve of the single-wavelength tapered diode laser system, the gain center of the tapered diode amplifier is around $801 \mathrm{~nm}$ [18]. Thus, the two wavelengths of the dual-wavelength diode laser system are tuned at each side of $801 \mathrm{~nm}$. The wavelength $\lambda_{1}$ is the shorter wavelength and $\lambda_{2}$ is the longer wavelength. The spectrum of the output beam from the dual-wavelength diode laser system is measured using a spectrum analyzer (Advantest Corp., Q8347) at different frequency differences between the two wavelengths. Figure 2 shows eight spectra with the frequency difference tuned from 0.57 to $10.02 \mathrm{THz}$ at an injected current of $3.5 \mathrm{~A}$. The suppression of the amplified spontaneous emission intensity is more than $25 \mathrm{~dB}$ for all the eight spectra, and the difference of the intensity of the two wavelengths measured in these spectra is less than $3 \mathrm{~dB}$. Compared to the results from the previous dualwavelength diode laser system [18], the frequency difference at the high-frequency end has been extended from 5.0 to 10.0 THz. But at the low-frequency end, like the condition in [18], the dual-wavelength operation with frequency difference less than $1.0 \mathrm{THz}$ is not stable, and the reason may be the competition and nonlinear interaction between the two wavelengths in the gain medium.

The output power of the dual-wavelength diode laser system at different frequency differences is shown in Fig. 3 at an operating current of $3.5 \mathrm{~A}$, and the corresponding two wavelengths are also shown in the figure. More than $1.6 \mathrm{~W}$ output power is obtained when the frequency difference is around $0.57 \mathrm{THz}$. The output power of the dual-wavelength diode laser system is higher than $0.95 \mathrm{~W}$ in the $10.0 \mathrm{THz}$ tuning range of the frequency difference of the two wavelengths. The output power decreases as the frequency difference of the two wavelengths increases, since both wavelengths of the dualwavelength diode laser system are tuned further away from

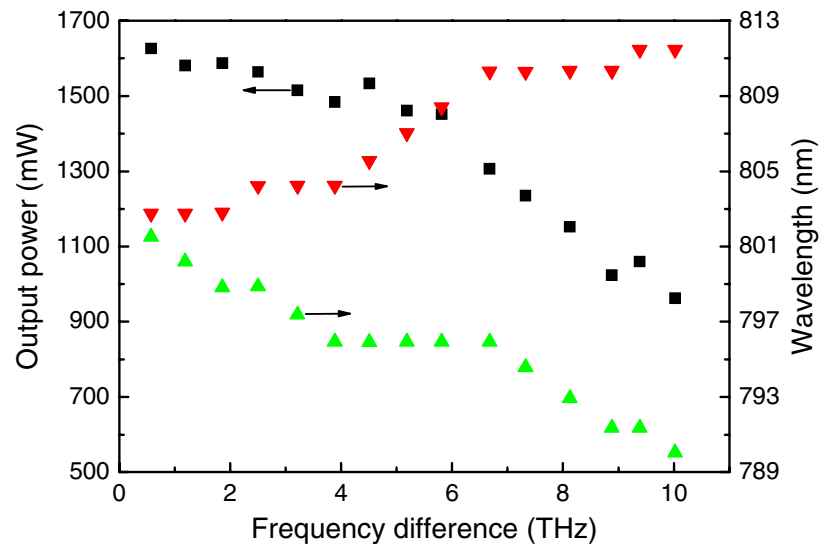

Fig. 3. (Color online) Output power from the dual-wavelength tapered diode laser system versus the frequency difference of the two wavelengths (squares), and the corresponding wavelength $\lambda_{1}$ (triangles) and $\lambda_{2}$ (inverted triangles).

the gain center of the tapered amplifier. The output power from the present dual-wavelength diode laser system is almost the same as that from the previous dual-wavelength diode laser system [18]; although in the previous laser system the cube $50 / 50 \mathrm{BS}$ in the external cavity introduced 50\% loss of the feedback beam, whereas in the present laser system the PBS does not introduce any loss. The reason of this fact is that the polarization directions of both feedback beams from gratings $G_{1}$ and $G_{2}$ are rotated around $45^{\circ}$ relative to their original polarization direction by the HWP; therefore, both feedback beams passing through the HWP are polarized around $45^{\circ}$ to the fast axis of the tapered amplifier. Since the optical component polarized along the fast axis of the tapered amplifier dominates the laser emission in the tapered amplifier, only around $50 \%$ of the feedback light power is amplified in the tapered amplifier; thus, the output power from the present dual-wavelength diode laser system is almost the same as that from the previous one.

The power stability of the dual-wavelength diode laser system at each wavelength is evaluated by measuring the power of each wavelength after the dual-wavelength output beam is dispersed by grating $G_{3}$ into two monochromatic beams. With a frequency difference of $5.64 \mathrm{THz}\left(\lambda_{1}=795.51 \mathrm{~nm}\right.$, $\left.\lambda_{2}=807.59 \mathrm{~nm}\right)$, the output powers for each wavelength

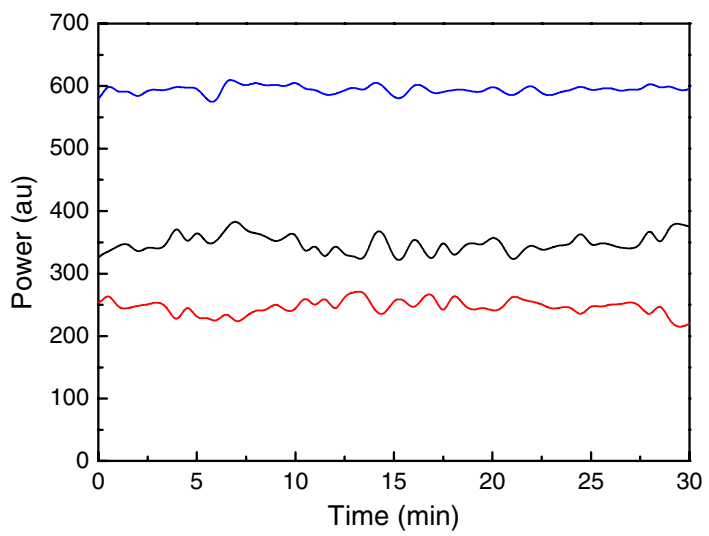

Fig. 4. (Color online) Measurement of the stability of the output power at $\lambda_{1}$ (black curve) and $\lambda_{2}$ (red curve) and the total output power (blue curve) from the dual-wavelength tapered diode laser system. 
are measured for $30 \mathrm{~min}$, and shown in Fig. 4. The output power fluctuations are $\pm 10.7 \%$ and $\pm 12.9 \%$ in 30 min for wavelength $\lambda_{1}$ and $\lambda_{2}$, respectively. Assuming that the diffraction efficiencies of the grating for both wavelengths are the same, the stability of the total output power of the dualwavelength diode laser system is the same as the stability of the sum of powers at each wavelength. The sum of powers at each wavelength is also shown in Fig. 4 , and it fluctuates in $\pm 3.8 \%$ in $30 \mathrm{~min}$. The fluctuation of the total power is much less than that of each of the two wavelengths. This means although dual-wavelength oscillation is achieved in the diode laser system, there is still competition between the two wavelengths, and the energy transfers between the two wavelengths back and forth. Thus, the output power fluctuations of the two wavelengths are almost of opposite phase. When using this laser system as a pump source for $\mathrm{THz}$ generation in a photomixer, the THz power will scale with the product of the optical powers of the two wavelengths [5]. The product of the optical powers of the two wavelengths is calculated, and it fluctuates in $\pm 8.6 \%$ in 30 min (i.e., the fluctuation of the generated $\mathrm{THz}$ power will be $\pm 8.6 \%$ in $30 \mathrm{~min}$ ). The fluctuation of the product of the powers of the two wavelengths is also much smaller than that of each of the two wavelengths, and the reason is the output power fluctuations of the two wavelengths are almost of opposite phase. We hope that the power fluctuation of each wavelength may be lowered by use of a more mechanically stable setup to half of the present value, and the fluctuation of the power of the generated $\mathrm{THz}$ radiation will be less than $\pm 4.0 \%$.

For the dual-wavelength external-cavity diode laser system, the beam quality of the output beam along the fast axis is assumed to be diffraction-limited because of the waveguide structure of the tapered device. The beam quality of the output beam along the slow axis is determined by measuring the beam quality factor $M^{2}$. A spherical lens with a $75 \mathrm{~mm}$ focal length is used to focus the diagnostic beam. The beam width, $W\left(1 / e^{2}\right)$, is measured at various recorded positions along the optical axis on both sides of the beam waist. The value of $M^{2}$ is obtained by fitting the measured data with a hyperbola. The average value of the two wavelengths is used as wavelength parameter in the fitting. Figure $\underline{5}$ shows the measured beam widths and the fitted curve with an output power of $1.45 \mathrm{~W}$, where the obtained $M^{2}$ value is $1.24 \pm 0.12$ with a $2.92 \mathrm{THz}$

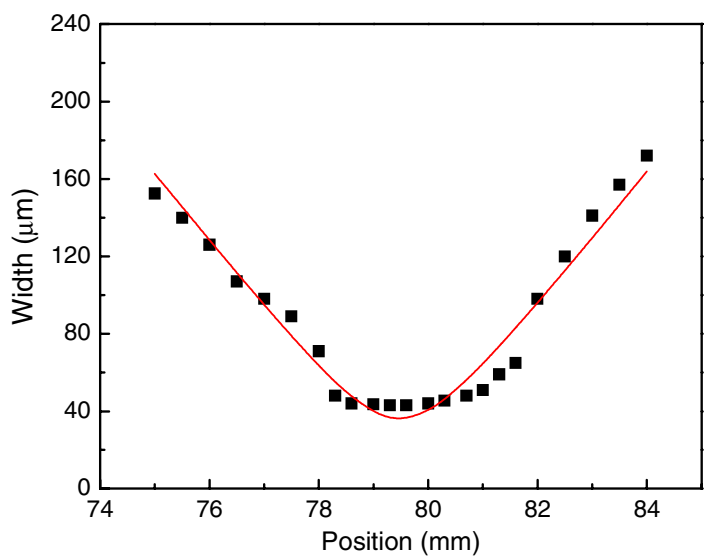

Fig. 5. (Color online) Beam width of the output beam in the slow axis with an output power of $1.45 \mathrm{~W}$ and a frequency difference of $2.92 \mathrm{THz}$. The curve represents a hyperbola fit to the measured data.

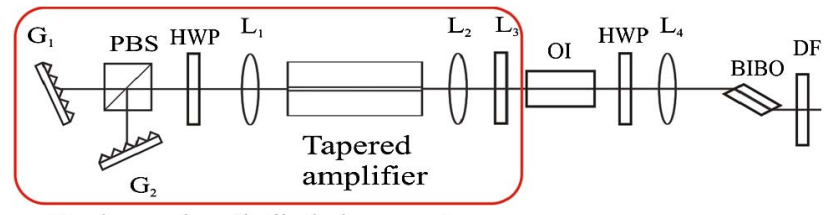

Dual-wavelength diode laser system

Fig. 6. (Color online) Experimental setup for the SFG experiment to test the simultaneous oscillation of the two wavelengths from the dual-wavelength diode laser system. $L_{1}, L_{2}$, aspherical lenses; $L_{3}$, cylindrical lens; $L_{4}$, biconvex lens; HWP, half-wave plate; PBS, polarized beam splitter; $G_{1}, G_{2}$, gratings; OI, optical isolator; $\mathrm{DF}$, dichroic filter.

$\left(\lambda_{1}=797.02 \mathrm{~nm}, \lambda_{2}=803.25 \mathrm{~nm}\right)$ frequency difference of the two wavelengths.

\section{SUM-FREQUENCY GENERATION IN A BIBO CRYSTAL}

The simultaneous emission of the two wavelengths in a dualwavelength diode laser system is important for many applications, e.g., a pump source for $\mathrm{THz}$ generation by difference frequency generation in a nonlinear crystal. The simultaneous emission of the two wavelengths in the diode laser system described above is verified by SFG in a BIBO crystal. The $5 \mathrm{~mm}$ long crystal with an aperture of $3 \mathrm{~mm} \times 3 \mathrm{~mm}$ is cut with $\theta=$ $151.9^{\circ}$ and $\varphi=90^{\circ}$ for type I phase matching (eeo) for frequency doubling at $808 \mathrm{~nm}$. The crystal is Brewster cut at $61.23^{\circ}$ to suppress the reflection at the end surfaces.

The experimental setup is shown in Fig. 6 . A $38 \mathrm{~dB}$ optical isolator is used to avoid the feedback from the optical components and the nonlinear crystal. A HWP is used to rotate the polarization direction of the beam to match the $e$-polarization direction (fast axis direction of the tapered amplifier) of the crystal, since the optical isolator rotates the polarization direction of the output beam from the diode laser system by $45^{\circ}$. A biconvex lens of $75 \mathrm{~mm}$ focal length is used to focus the beam into the BIBO crystal. A dichroic filter separates the signal from the infrared pump beam.

The two wavelengths of the pump beam from the dualwavelength diode laser system are tuned to 796.81 and $802.83 \mathrm{~nm}$ (the frequency difference is $2.82 \mathrm{THz}$ ). By rotating

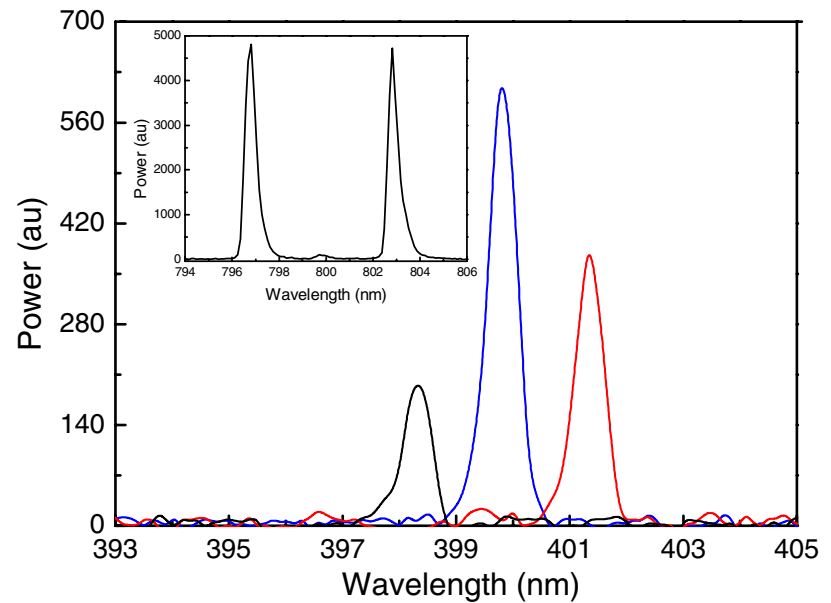

Fig. 7. (Color online) Optical spectra of the second-harmonic signals of each of the two wavelengths (black and red curves) and sumfrequency signal of the two wavelengths (blue curve). Inset: spectrum of the infrared pump beam from the dual-wavelength diode laser system. 
the BIBO crystal, three signals in the UV range are observed at three different incident angles of the pump beam. The spectra of the three signals in the UV range are recorded using a spectrometer (Ocean Optics Corp. HR4000) and shown in Fig. 7. These three signals correspond to the two second-harmonic signals of each of the two wavelengths and a sum-frequency signal of the two wavelengths. The spectrum of the pump beam from the dual-wavelength diode laser system is shown in the inset in Fig. 7. The observed sum-frequency signal means that the two wavelengths emit simultaneously.

\section{CONCLUSION}

A dual-wavelength diode laser system based on a tapered amplifier with double-Littrow external cavity is demonstrated around $800 \mathrm{~nm}$. The frequency difference of the two wavelengths is tunable from 0.5 to $10.0 \mathrm{THz}$. To our knowledge, this is the broadest tuning range of the frequency difference from a dual-wavelength diode laser system. The output power is higher than $0.95 \mathrm{~W}$ in the entire tuning range of frequency difference, and a maximum power of $1.6 \mathrm{~W}$ is obtained with a frequency difference of $0.57 \mathrm{THz}$. The stability of the output power at each wavelength is measured, and the power fluctuations of the two wavelengths are of opposite phase. With a frequency difference of $2.92 \mathrm{THz}$, an $M^{2}$ value of 1.24 in the slow axis is determined with an output power of $1.45 \mathrm{~W}$. The simultaneous oscillation of the two wavelengths is verified by the SFG experiment in a BIBO crystal.

\section{ACKNOWLEDGMENT}

The authors acknowledge Bernd Sumpf and Götz Erbert from Ferdinand-Braun-Institut, Leibniz-Institut für Höchstfrequenztechnik for the tapered diode amplifier.

\section{REFERENCES}

1. P. Groot, "Use of a multimode short-external-cavity laser diode for absolute-distance interferometry," Appl. Opt. 32, 4193-4198 (1993).

2. M. Tani, P. Gu, M. Hyodo, K. Sakai, and T. Hidaka, "Generation of coherent terahertz radiation by photomixing of dual-mode lasers,” Opt. Quantum Electron. 32, 503-520 (2000).

3. T. Kleine-Ostmann, P. Knobloch, M. Koch, S. Hoffmann, M. Breede, M. Hofmann, G. Hein, K. Pierz, M. Sperling, and K. Donhuijsen, "Continuous-wave THz imaging," Electron. Lett. 37, 1461-1463 (2001).

4. S. Hoffmann, M. Hofmann, M. Kira, and S. W. Koch, "Two-colour diode lasers for generation of THz radiation," Semicond. Sci. Technol. 20, S205-S210 (2005).

5. I. Park, C. Sydlo, I. Fischer, W. Elsäßer, and H. L. Hartnagel, "Generation and spectroscopic application of tunable continuous-wave terahertz radiation using a dual-mode semiconductor laser," Meas. Sci. Technol. 19, 065305 (2008).

6. T. Hidaka, S. Matsuura, M. Tani, and K. Sakai, "CW terahertz wave generation by photomixing using a two-longitudinal-mode laser diode," Electron. Lett. 33, 2039-2040 (1997).

7. M. Al-Mumin, C. Kim, I. Kim, N. Jaafar, and G. Li, "Injection locked multi-section gain-coupled dual mode DFB laser for terahertz generation," Opt. Commun. 275, 186-189 (2007).

8. A. Klehr, J. Fricke, A. Knauer, G. Erbert, M. Walther, R. Wilk, M. Mikulics, and M. Koch, "High-power monolithic two-mode DFB laser diodes for the generation of THz radiation," IEEE J. Sel. Top. Quantum Electron. 14, 289-294 (2008).

9. N. Kim, J. Shin, E. Sim, C. W. Lee, D. Yee, M. Jeon, Y. Jang, and K. H. Park, "Monolithic dual-mode distributed feedback semiconductor laser for tunable continuous-wave terahertz generation," Opt. Express 17, 13851-13859 (2009).

10. N. Kim, S. Han, H. Ko, Y. A. Leem, H. Ryu, C. W. Lee, D. Lee, M. Y. Jeon, S. K. Noh, and K. H. Park, "Tunable continuous-wave terahertz generation/detection with compact $1.55 \mu \mathrm{m}$ detuned dualmode laser diode and InGaAs based photomixer," Opt. Express 19, 15397-15403 (2011).

11. S. Hoffmann, M. Hofmann, E. Bründermann, M. Havenith, M. Matus, J. V. Moloney, A. S. Moskalenko, M. Kira, S. W. Koch, S. Saito, and K. Sakai, "Four-wave mixing and direct terahertz emission with two-color semiconductor lasers," Appl. Phys. Lett. 84, 3585-3587 (2004).

12. I. Park, I. Fischer, and W. Elsäßer, "Highly nondegenerate fourwave mixing in a tunable dual-mode semiconductor laser," Appl. Phys. Lett. 84, 5189-5191 (2004).

13. W. Wang, M. Cada, J. Seregelyi, S. Paquet, S. J. Mihailov, and P. Lu, "A beat-frequency tunable dual-mode fiber-Bragg-grating externalcavity laser," IEEE Photon. Technol. Lett. 17, 2436-2438 (2005).

14. V. Zambon, M. Piché, and N. McCarthy, "Tunable dualwavelength operation of an external cavity semiconductor laser," Opt. Commun. 264, 180-186 (2006).

15. C. Friedrich, C. Brenner, S. Hoffmann, A. Schmitz, I. C. Mayorgak, A. Klehr, G. Erbert, and M. R. Hofmann, "New two-color laser concepts for THz generation," IEEE J. Sel. Top. Quantum Electron. 14, 270-276 (2008).

16. S. A. Zolotovskaya, N. Daghestani, G. B. Venus, L. B. Glebov, V. I. Smirnov, and E. U. Rafailov, "Stable dual-wavelength operation of InGaAs diode lasers with volume Bragg gratings," Appl. Phys. Lett. 91, 171113 (2007).

17. S. A. Zolotovskaya, V. I. Smirnov, G. B. Venus, L. B. Glebov, and E. U. Rafailov, "Two-color output from InGaAs laser with multiplexed reflective Bragg mirror," IEEE Photon. Technol. Lett. 21, 1093-1095 (2009).

18. M. Chi, O. B. Jensen, and P. M. Petersen, "High-power dualwavelength external-cavity diode laser based on tapered amplifier with tunable terahertz frequency difference," Opt. Lett. 36, 2626-2628 (2011).

19. B. Sumpf, K. Hasler, P. Adamiec, F. Bugge, F. Dittmar, J. Fricke, H. Wenzel, M. Zorn, G. Erbert, and G. Tränkle, "High-brightness quantum well tapered lasers," IEEE J. Sel. Top. Quantum Electron. 15, 1009-1020 (2009).

20. M. Chi, O. B. Jensen, J. Holm, C. Pedersen, P. E. Andersen, G. Erbert, B. Sumpf, and P. M. Petersen, "Tunable high-power narrowlinewidth semiconductor laser based on an external-cavity tapered amplifier," Opt. Express 13, 10589-10596 (2005). 